\title{
Le renard de Rutebeuf
}

\section{Aurélie Barre}

\section{OpenEdition}

Journals

Édition électronique

URL : https://journals.openedition.org/crm/2685

DOI : 10.4000/crm.2685

ISSN : 1955-2424

\section{Éditeur}

Honoré Champion

\section{Édition imprimée}

Date de publication : 15 décembre 2007

Pagination : 253-266

ISSN : 1272-9752

\section{Référence électronique}

Aurélie Barre, "Le renard de Rutebeuf », Cahiers de recherches médiévales [En ligne], 14 | 2007, mis en ligne le 15 décembre 2010, consulté le 15 décembre 2022. URL : http://journals.openedition.org/crm/ 2685 ; DOI : https://doi.org/10.4000/crm.2685 


\title{
酮RM
}

\section{Le renard de Rutebeuf}

\author{
[...] La clarté de sa prose et ses discours si \\ bien entortillés lui semblaient des perles et surtout \\ quand il venait à lire ces lettres de galanterie et de défi \\ où il trouvait écrit en plus d'un endroit: La raison de \\ la déraison qu'à ma raison vous faites affaiblit \\ tellement ma raison que, avec raison, je me plains de \\ votre beauté ${ }^{1}$.
}

Rutebeuf ${ }^{2}$ regarde le monde: les architectures sont en ruines et les objets brisés, les arbres perdent leurs feuilles et l'hiver s'installe. "Li vers" du poète évoquent ce monde "divers ", à la fois changeant et mauvais, et "l'ivers", la froide saison du dénuement et de la mort ${ }^{3}$. De nouvelles valeurs règlent alors les conduites : l'argent a remplacé la générosité ; Avarice, Cruauté et Envie ont mis à mal Humilité, Charité, Noblesse d'Âme. Alors que les villes et les universités se développent, une théologie nouvelle imprègne les rues et les esprits. L'univers décrit par Rutebeuf est instable et perverti ; les agents de ce monde «bestourné » sont les frères mendiants. Dans l'imaginaire du poète, ils sont régulièrement assimilés au renard, symbole de la ruse et de l'hypocrisie.

L'activité du poète trouve son origine dans ce monde en rupture avec les valeurs anciennes, courtoises et épiques : "Rimeir me covient de cest monde / Qui de touz biens ce wide et monde $»^{4}$. Et, sous maintes formes poétiques, avec toutes les ressources de la rhétorique, la parole de Rutebeuf révèle, dénonce, menace, sur un ton de déploration ou de satire. L'écriture du poète tente à son tour de retourner le monde inversé, de le remettre à l'endroit, dans le sens des règles et des valeurs religieuses et morales. Et puisque le monde est bestourné, il va en donner une image littérale et le "tourner en bête»: les hommes hypocrites sont des renards. Ainsi malgré leur froc gris ceint d'une corde, derrière leurs masques, les mendiants s'appellent Renart5.

Comme par un échange de qualité, la parole de satire et de dénonciation utilise les codes et les masques du monde nouveau, elle se déguise. Rutebeuf se fait alors lui-même renard pour mieux déjouer les pièges du monde et se laisse séduire par la beauté des artifices; sa poétique est renardienne.

\footnotetext{
${ }^{1}$ Cervantès, Don Quichotte, Paris, Gallimard, «Folio classique », 2006, t. 1, p. 68.

${ }^{2}$ Les références de cet article renvoient toutes aux Euvres complètes de Rutebeuf, texte établi, traduit, annoté et présenté par M. Zink, Paris, Le Livre de Poche, Classiques Garnier, «Lettres gothiques », 2002.

${ }^{3}$ Voir par exemple La Griesche d'hiver (v. 6-8) ou La Complainte Rutebeuf (v. 79-81), ibid., p. 196 et 322.

${ }^{4}$ Les Plaies du monde, ibid., p. 72, v. 1-2.

${ }^{5}$ Voir la laisse VII de La Discorde des Jacobins et de l'Université, ibid., p. 120.
}

Cahiers de Recherches Médiévales, 14, 2007 


\section{Hypocrites renards}

Avant d'être Renart, le personnage hérité du Roman de Renart, le renard de Rutebeuf est un goupil rusé, l'animal sauvage régulièrement décrit par les livres de chasse et les Bestiaires. La ruse de l'animal, sa fourberie à l'égard des oiseaux, est rappelée dans le prologue du Dit des règles, alors que Rutebeuf compare les frères mendiants au goupil :

\footnotetext{
Ausi nos prennent et desoivent

Com li werpyz fait les oiziaux.

Saveiz que fait li damoiziaus?

En terre rouge se rooille,

Le mort fait et la sorde oreille,

Si viennent li oizel des nues,

Et il ainme mout lor venues,

Car il les ocist et afole. (v. 10-15)
}

L'ancien nom commun du renard, «werpyz», rappelle l'usage des encyclopédies médiévales; dans le poème de Rutebeuf, le détail de la terre rouge dont le renard recouvre ses poils provient du Physiologus Latinus ${ }^{6}$ :

Uulpis est animal dolosum et nimis fraudulentum et argumentorum. Cum esurierit et non inuenerit quod manducet, requirit locum ubi est rubra terra, et voluit se super eam, ita ut quasi cruenta appareat tota; et proicit se in terram, et uoluit se super eam tamquam mortua; et, attrahens intra se flatus suos, ita se inflat ut penitus non respiret. Aves uero uidentes eam sic inflatam et quasi cruentam iacentem, et linguam eius aperto ore foris eiectam, putant eam esse mortuam; et descendunt et sedent super eam : illa uero rapit eas et deuorat.

Le Bestiaire de Pierre de Beauvais précise lui aussi que le renard «se roule dans la terre rouge de telle sorte qu'il donne l'impression d'être tout ensanglanté »? Dans le Bestiaire divin de Guillaume le Clerc de Normandie, sur la miniature qui ouvre la section «Renard», l'animal contrefait le mort. Sa pelisse rougie suggère qu'il s'est roulé dans la terre argileuse sur laquelle il est étendu'.

La ruse est d'abord fortement animalisée. Dans le prologue du Dit des règles, le renard est bien le goupil. Mais, immédiatement après avoir utilisé le substantif «werpyz», au vers suivant, Rutebeuf substitue l'homme à l'animal: le renard devient «li damoiziaus », possible image des hommes et plus précisément, dans ce dit, des hypocrites (v. 33) et des ordres mendiants à peine nommés. Car le substantif désigne certes au Moyen Âge le jeune homme noble, l'apprenti chevalier mais aussi le courtisan qui use de flatteries et de douces manières pour obtenir ce qu'il convoite.

\footnotetext{
${ }^{6}$ Physiologus Latinus, édité par F. J. Carmody, Paris, Droz, 1939, p. 29.

${ }^{7}$ Dans Bestiaires du Moyen Âge, mis en français moderne et présentés par G. Bianciotto, Paris, Stock, « Moyen Âge », 1980, p. 37-38.

${ }^{8}$ Guillaume le Clerc, Bestiaire divin, Lyon, BM, ms. Palais des Arts 78, fol. $41^{\mathrm{v} .}$
} 
L’imaginaire de Rutebeuf, hérité des Bestiaires, est également conditionné par la tradition du Roman de Renart où déjà la feinte et le déguisement permettent de relier l'hypocrite, le religieux et le renard. Étymologiquement, hupocrisis est un terme de théâtre : il désigne une réplique puis, plus généralement, le jeu de l'acteur, la feinte, les faux-semblants. L'hypocrite devient celui qui déguise ses pensées et qui séduit par ses paroles et ses mines trompeuses. Le renard du Physiologus teinte sa pelisse de terre rouge; dans la branche Ic du Roman de Renart, le goupil prend un accent anglais et arbore une éclatante couleur jaune grâce à laquelle il trompe et abuse Isengrin; il se frotte le visage et le corps d'une poudre noire dans «Renart le noir» (v. 1209-1212) ${ }^{9}$. Dans plusieurs de ses branches, Renart fait le mort pour attraper des oiseaux ${ }^{10}$ ou tromper les marchands ${ }^{11}$. Il manipule aussi adroitement la dévotion $^{12}$ et connaît le langage sacré qu'il travestit aisément ${ }^{13}$.

L'art de la dissimulation et de la déception trouve un écho dans plusieurs dits de Rutebeuf: les frères mendiants cachent l'orgueil et la cupidité sous leur robe grossière, sous l'apparente simplicité de leur mise et de leur visage ${ }^{14}$. "Par maint samblant, par mainte guisse», "par fausse samblance ${ }^{15}$, ils investissent la ville et prêchent leurs règles. Par l'intermédiaire de proverbes, Rutebeuf met en garde les hommes contre ce jeu diabolique des apparences: "N'est pas tot ors quanque reluit $»^{16}:$ : Li abiz ne fait pas l'ermite $»^{17}$.

Comme les proverbes, les images du simulacre et de la dissimulation du diabolique potentiellement actif sont prégnantes. Le feu couve dans la Complainte de Guillaume de Saint-Amour ${ }^{18}$; la braise persiste sous la cendre dans la Leçon sur Hypocrisie et Humilité :

Ce vous saveiz raison entendre,

C'est li charbons desoz la cendre,

Qui est plus chauz que cil qui flame. (v. 263-265)

La douce fleur masque l'épine dans Le Dit des Jacobins :

\footnotetext{
${ }^{9}$ Toutes les références au Roman de Renart renvoient à l'édition dirigée par A. Strubel, Paris, Gallimard, «Bibliothèque de la Pléiade », 1998.

${ }^{10}$ Voir « Renart le noir» (v. 902-926) et « La Mort de Renart» (v. 1430-1454), ibid.

${ }^{11}$ Voir « Renart et les anguilles» ou « Renart et Primaut», ibid.

${ }^{12}$ Voir par exemple «Les Vêpres de Tibert», ibid.

${ }^{13}$ Dans « Le Puits », Renart évoque pour éveiller le désir d'Isengrin, l'abondance de nourriture que recèle le paradis, ibid.

${ }^{14}$ Voir le jeu d'annominatio à partir du mot «simple» dans D'Hypocrisie, Rutebeuf, Euvres complètes, éd. cit., p. 138, v. 51-55.

${ }^{15}$ Ces expressions sont présentes dans les Ordres de Paris, ibid., p. 246, v. 13 et 19.

${ }^{16}$ Sur l'hypocrisie (Du Pharisien), Ibid., p. 140, v. 92. Le proverbe répertorié par Morawski (1371) est très régulièrement utilisé par Rutebeuf. Voir Le Dit de frère Denise le cordelier (v. 15), La Complainte de Guillaume de Saint-Amour (v.21), Le Miracle du Sacristain (v. 428), La Vie de Sainte Elysabel (v. 654) ou La Complainte d'Outremer (v. 38).

${ }^{17}$ Le Dit de Frère Denise le cordelier, ibid., v. 1.

${ }^{18}$ «Com plus couve li fex, plus art», ibid., v. 29.
} 
Car teiz vest rude robe ou felons cuers repouze :

Li roziers est poignans et c'est soeiz la roze. (v. 47-48)

ou dans La Discorde des Jacobins et de l'Université:

Se lor huevre ne se concorde

A l'abit qu'ameir Dieu devise,

Au recordeir aura descorde

Devant Dieu au jor dou Juÿse.

Car ce Renart seint une corde

Et vest une coutele grise,

N'en est pas sa vie mains orde :

La roze est sus l'apine asize. (v. 49-56)

La rose cache l'épine comme la corde des mendiants l'ordure: dans cette laisse, Rutebeuf fait partout résonner «orde». Le mot s'entend à la rime dans « concorde», «descorde» et « corde», mais aussi au début du vers 51 dans « recordeir». Comme la métaphore, l'annominatio resémantise les mots dont l'apparence positive, le sens originel, masque en réalité l'ordure. Et, au milieu de ces images et de ces jeux rhétoriques, survient Renart (v. 53) : puisque les frères sont des hypocrites, alors le poète les assimile à Renart ${ }^{19}$ et dénonce l'être dissimulé sous le paraître.

Chez Rutebeuf, dans La Discorde des Jacobins et de l'Université ou dans D'Hypocrisie (ou Du Pharisien), l'hypocrite s'appelle donc Renart ${ }^{20}$. L'histoire du nom propre est explicitée dans la branche XXV du Roman de Renart. «Les Enfances de Renart» est une réécriture parodique de la Genèse : Adam crée les animaux domestiques alors qu'Ève donne successivement naissance au loup et au renard. Le narrateur révèle alors la signification du nom propre «Renart» qui remplacera « goupil ${ }^{21}$ :

\author{
Ici gorpis vos senefie \\ Renars, qui tant sot de minstrie \\ Tot cil qui sont d'anging et d'art, \\ Sunt mes tuit apelez Renart \\ Por Renart et por son gorpil. (v. 101-105) 22
}

Le renard dissimule la réalité sous des simulacres, maîtrise à la perfection la ruse et l'art de la semblance. Tous ceux qui manipulent les signes et les artifices portent donc son nom (goupil ou renard/t).

L'écriture allégorique, dans D'Hypocrisie, comme le détour par le nom et la bête, permet une dénonciation et une satire très fortes de la religion, elle révèle le piège des mots et des apparences : le masque, dont l'allégorie, la personnification ou

\footnotetext{
${ }^{19}$ Dans le même dit, à la laisse VI, les frères sont comparés au loup (v. 45-48), ibid.

${ }^{20} \mathrm{Au}$ vers 80 , le poète nous met en garde contre «Ypocrisie la renarde », ibid.

${ }^{21}$ Les premières attestations du nom commun «renard» datent du XIII ${ }^{\mathrm{e}}$ siècle. L'emploi le plus ancien est relevé chez Gautier de Coincy en 1223.

${ }_{22}^{2}$ Les Enfances de Renart», Le Roman de Renart, éd. cit., p. 829.
} 
le symbole sont les procédés littéraires, a vocation, par ironie, à dénoncer les fauxsemblant et, chez Rutebeuf, comme plus tard dans le Tartuffe de Molière, la relation très privilégiée, et pourtant antithétique, qui se noue entre hypocrisie et religion. Pour les théologiens en effet, l'hypocrisie est un péché mortel; elle est stigmatisée par la Bible, en particulier dans l'Évangile de Matthieu :

Malheur à vous, scribes et Pharisiens hypocrites, qui ressemblez à des sépulcres blanchis: au-dehors ils ont belle apparence, mais au-dedans ils sont pleins d'ossements de morts et de toute pourriture ; vous de même, au-dehors vous offrez aux yeux des hommes l'apparences des justes, mais au-dedans vous êtes pleins d'iniquité et d'hypocrisie » (Matth., 23, 27-28).

Hypocrites, les frères mendiants ont donc détourné les règles, ils ont inversé les valeurs morales et théologiques, ils ont aussi bestourné le monde. Le verbe signifie tout à la fois «tourner à l'envers, renverser, détruire, tourner mal voire déguiser». Il est employé dans Le Dit de l'Université de Paris (v. 24) et dans L'État $d u$ monde (v. 84), où il prend le sens d'inverser, les clercs avaricieux mettant l'argent avant la justice. Ce renversement des valeurs rappelle les fêtes des fous et les carnavals : le temps de la fête, de l'exubérance et des débordements, le profane remplace le sacré, la société se régale de ses péchés. Le Testament de l'âne illustre presque une fête de l'âne ${ }^{23}$ où le riche (le «foz», v. 123) rend hommage au plus faible, l'âne, en le faisant son héritier. Mais les dits sont plus sombres que ce petit conte proche du fabliau: les mendiants excèdent mille fois le péché du prêtre cupide.

Dans ses brèves apparitions, le renard de Rutebeuf, symbole de la ruse, de la dissimulation et de l'hypocrisie constitue ainsi l'image animale des moines mendiants. Le renard n'est donc plus le fin goupil des Bestiaires ou du Roman de Renart, habile à duper les autres pour parvenir à remplir son ventre creux, son art est littéralement diabolique. Pareils à Renart, qui manipule les signes, déguise sa pelisse de jaune ou de noir et son langage de quelques couleurs de rhétorique, les mendiants établissent une différence entre le signe et son référent, entre l'apparence et l'essence. La duplicité, la perversion des signes présente à la fois dans leur allure et dans leur rhétorique trompeuse, leurs «blanches paroles $»^{24}$, relèvent de la «diabolie $»^{25}$.

\section{Renart le bestourné}

Le renard n'était jusque-là dans la poétique de Rutebeuf qu'une présence furtive, une image fuyante et impromptue tout à la fois de la ruse, de l'hypocrisie et

\footnotetext{
${ }^{23}$ Voir J. Heers, Fêtes des fous et carnavals, Paris, Fayard, «Pluriel », 1983 et en particulier les pages 136 à 141 consacrées à la fête de l'âne.

${ }^{24}$ Dans cette expression empruntée au Roman de Renart, «blanches» est certainement la déformation de «blandes ». Le latin blandus signifie « flatteur, séduisant».

${ }^{25}$ À l'origine de « diable », le mot provient du grec «diabolos» : « qui désunit » et s'oppose au «sumbolon»: «mettre ensemble, réunir, harmoniser». Sur la diabolie dans le Roman de Renart, voir l'ouvrage de Cl. Reichler: La Diabolie. La séduction, la renardie, l'écriture, Paris, Les Éditions de Minuit, «Critique », 1979, p. 77-149.
} 
du Mal, la représentation symbolique des mendiants. Dans le dit de Renart le Bestourné, le renard devient une véritable incarnation envahissante du Mal et le récit satirique figure pleinement le renversement, le «bestournement» du monde.

Le poème de Rutebeuf semble à première vue largement puiser son matériau dans le Roman de Renart: il lui emprunte ses personnages, ses tournures et ses images. L'intertexte renardien ne cesse donc de circuler dans la pièce: Renart appartient au royaume de Noble le lion, image animale du roi Louis XI et il chevauche un destrier (v. 5) comme lorsque, dans le Roman, il quitte la charrette des marchands ou entend lancer une attaque contre une abbaye cistercienne ${ }^{26}$. La branche intitulée «Renart empereur $~_{27}$ semble avoir en partie inspiré le poète : elle raconte «si con Renart fut emperieres par engin $»^{28}$, Renart ayant en effet profité de l'absence de Noble, parti défendre le royaume menacé par les Infidèles, pour usurper son pouvoir.

Mais Rutebeuf «bestourne» la tradition du Roman de Renart, il l'inverse en annulant la solitude essentielle du goupil rejeté au ban de la société, à Maupertuis. C'est désormais lui qui règne sur le royaume et qui a autorité sur le roi ; ses ennemis d'hier, Isengrin, Ronel et Bernard, sont devenus ses alliés. Dans le dit de Renart le Bestourné, l'étendue du pouvoir de Renart sur la société est mimée par son omniprésence dès les trois premiers vers de la pièce ${ }^{29}$ :

Renars est mors : Renars est vis !

Renars est ors, Renars est vilz :

Et Renars reigne! (v. 1-3)

À chaque fois sujet des verbes, force agissante, Renart est immortel, partout réincarné là où se trouvent la pourriture, la vilenie et l'hypocrisie révélée par la syllepse : «ors» signifiant à la fois «l'ordure » et «l'or» qui la dissimule. Les jeux rhétoriques de Rutebeuf n'ont de cesse de démultiplier le sens des mots : les rimes et les échos redisent l'abject et sa permanence ${ }^{30}$. Le vers qui clôt le premier tercet de la pièce, plus court que les deux autres, assène la vérité de l'omnipotence du goupil : le

\footnotetext{
${ }^{26}$ Dans « Renart et les anguilles», les marchands ne parviennent par à rattraper le goupil car, précise le conteur : «il a trop isnel cheval» (v. 143); dans « Le Puits », Renart est impatient de lancer un assaut contre le poulailler: "Onques ne fu ses frains tenus / Tant qu'il est as capons venus»(v. 87-88), Le Roman de Renart, éd. cit. Sur ce sujet, voir l'article de G. Bianciotto : «Renart et son cheval », Mélanges Félix Lecoy, Paris, Champion, 1973, p. $27-$ 42 ; B. Ribémont, «Renart et le cheval», Reinardus, 7, 1994, p. 127-42.

${ }^{27}$ «Renart empereur» est la branche XVI du ms H (Le Roman de Renart, éd. cit., p. 563-645).

${ }^{28}$ Avant le début de la branche, le copiste du manuscrit $M$ insère une rubrique : « $\mathrm{Ci}$ faut si conme Renart fu mires et conmence si con Renart fut emperieres par engin ».

${ }^{29}$ Voir J. Dufournet, «Sur la structure des vingt-et-un premiers vers de Renart le Bestourné », L'Univers de Rutebeuf, op. cit., p. 61-65. Voir aussi l'article de R. Dragonetti : «Renart est mort, Renart est vif, Renart règne», La Musique et les lettres: études de littérature médiévale, Genève, Droz, «Publications romanes et françaises », p. 419-434.

${ }^{30}$ La mention de l'or fait peut-être référence à «Renart teinturier », Le Roman de Renart, éd. cit., branche Ic.
} 
« $\mathrm{r} »$ de Renart consonne d'ailleurs avec celui de « reigne », comme si les mots étaient équivalents.

Le poète traduit ainsi l'invasion du Mal qui gouverne le nouveau royaume soumis aux lois de la ruse et de la perfidie. Avec Rutebeuf, le renard est devenu une figure politique et religieuse. L'image de l'animal est plus noire que dans les récits antérieurs, elle est fortement liée à la vision biblique : le renard y est une incarnation du diable ou de Judas, dont il a la couleur rousse ${ }^{31}$, et plus généralement le symbole des hérétiques. Le renard est la destruction, dans Le Livres des Juges lorsque Samson brûle les moissons des Philistins (15, 1-5), et le châtiment. Les renards ravagent les vignes au début du Cantique des Cantiques:

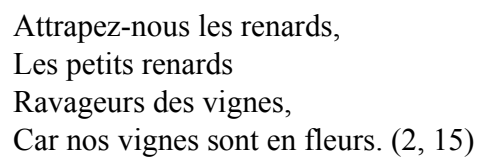

Grégoire le Grand insiste sur l'identité du renard et du diable ${ }^{32}$. Comme chez Ambroise $^{33}$, il assimile aussi l'animal aux hérétiques ${ }^{34}$. Pour Raban Maur, «le renard signifie allégoriquement le diable trompeur, les hérétiques rusés ou les pécheurs $»^{35}$.

Cette représentation du Mal imprègne donc progressivement la littérature après le Roman de Renart. L' «enging » et l'art du goupil se sont mués en une ruse maléfique; dans le même temps, la séduction exercée par l'animal dans le Roman et les fables a évolué vers une forte répulsion. Le goupil rusé devient pour les récits épigones une image du diable ${ }^{36}$.

Le premier de ces épigones est un texte poétique inséré dans les Mémoires en prose de Philippe de Novare, rédigés entre 1252 et 1257. Philippe de Novare reprend les personnages (jusqu'aux noms des fils de Renart conformes à la tradition) et la forme du Roman : l'octosyllabe à rimes plates. Il rappelle certains motifs du Roman de Renart: le retour auprès d'Hermeline à Maupertuis, la feinte de mort, la confession sans repentir, le "compissage» des louveteaux; il emprunte même quelques formules à la branche I du Roman. Philippe de Novare compose, c'est le mot qu'il emploie, une nouvelle branche: «La branche dit ensy: C'est la rime de Renart, come Yzengrin le desconfist $»^{37}$; la formule en prose qui précède le récit en

\footnotetext{
${ }^{31}$ Cette couleur qualifie les hypocrites dans La Complainte de maître Guillaume de SaintAmour: "Mais cil qui ont fauve la face», v. 92 (Rutebeuf, Euvres complètes, éd. cit., p. 158). Elle permet de rapprocher la tradition de Rutebeuf du Roman de Fauvel.

${ }^{32}$ Grégoire le Grand, Morales sur Job, P.L. LXXVI, 96.

${ }^{33}$ Par exemple dans Exposit. evang. Luc, VII, 31, 47, P.L. XV, 1707.

${ }^{34}$ Grégoire le Grand, Super Cant. Cantic. Exp. II, 17, P.L. LXXIX, 500 B-C.

${ }^{35}$ Raban Maur, De universo, VIII, 1. J. Voisennet renvoient à plusieurs textes des Pères et des auteurs médiévaux, Bêtes et hommes dans le monde médiéval. Le bestiaire des clercs $d u V^{e}$ au XII siècle, Turnhout, Brepols, 2000, p. 78-79 (notes).

${ }^{36}$ Sur les liens entre Rutebeuf et Le Roman de Renart, voir J. Dufournet: «Rutebeuf et le Roman de Renart », L'Univers de Rutebeuf, Orléans, Paradigmes, «Medievalia », 2005, p. 4160.

${ }^{37}$ La pièce satirique rimée est éditée dans Le Roman de Renart, éd. cit., p. 845-851.
} 
octosyllabes rappelle les rubriques présentes au début des branches dans le manuscrit $C^{38}$.

Mais, si le récit de Philippe de Novare, comme celui de Rutebeuf, est une profonde satire politique et sociale, où les hommes sont dissimulés sous des traits animaux ${ }^{39}$, son renard est bien plus proche de celui du Roman. Renart y symbolise essentiellement la ruse: «Mais tout ce est engin et art!» (v. 95). Il est plein de «lecherie » et de «trecherie » (v. 101-102), «de barat et de mal art» (v. 215). Renart le «trecheour» (v. 120), le «trechiere plumés» (v. 99) est un décepteur, non une image du Mal, comme chez Rutebeuf. Malgré la fin du texte («Dïables ot en luy grand part», v. 216), le récit ne fait pas du renard une personnification du Mal: il privilégie encore sa qualité d'animal fourbe et rusé.

Au-delà de 1261, le renard de Rutebeuf «at mout grant norreture» (v. 25 ${ }^{40}$. Car d'autres récits épigones, composés pour la plupart à la fin $\mathrm{du} \mathrm{XIII}$ siècle et très certainement influencés par Renart le Bestourné, noircissent davantage encore le renard. Plusieurs auteurs s'inspirent de cette pièce et accentuent le degré de personnification et d'exemplarité du personnage ${ }^{41}$ : ils identifient le renard au moine mendiant, hypocrite et maléfique ou en font une image allégorique: il est la Renardie, l'incarnation du langage spécieux et séducteur, qui captive et déçoit.

Dans Le Couronnement de Renart (1263) ${ }^{42}$, Renart a pris deux robes : celle des Franciscains et celle des Jacobins auxquels il enseigne ses ruses (v. 1174-1253) ${ }^{43}$ avant de se rendre à la cour de Noble pour prendre la couronne. La diabolisation de Renart se réalise pleinement dans la figure allégorique de Renardie qui désormais accompagne Orgueil et Envie :

Envie, Orgius et Renardie

Les bons a morir nos avie.

Renardie, Envie et Orgius

Les maus retient, s'ochit le mius. (v. 2765-2768)

\footnotetext{
${ }^{38}$ Voici quelques unes de ces rubriques : «Si conme Renart fist Ysangrin moine », «C'est si conme Renart fu tainturiers », «C'est le songe Renart si conme Ysangrin le bati »...

${ }^{39}$ Pour un commentaire plus historique et politique de ces masques, voir la riche notice de S. Lefèvre dans Le Roman de Renart, éd. cit., p. 1386-1393.

${ }^{40}$ « Norreture» prend ici le sens de « descendance, famille».

${ }^{41}$ Sur la proximité entre Renart et l'allégorie, voir le chapitre d'A. Strubel : «Les avatars de Renart» dans La Rose, Renart et le Graal. La littérature allégorique en France au XIII siècle, Paris-Genève, Slatkine, 1989, p. 229-243.

${ }^{42}$ Le Couronnement de Renart est édité par D. M. Méon dans le tome IV de son Roman de Renart (Paris, Treultel et Würtz, 1826). Il est partiellement édité dans Le Roman de Renart, éd. cit., p. 869-896.

${ }^{43}$ Le frère jacobin qui accompagne Renart à la cour voit clair dans son jeu : «Tant m'a apris dou renardet, / Puis que te vis premierement, / Que se verités ne me ment / Jou te cuit coroner a roi. » (Le Couronnement de Renart, édité dans Le Roman de Renart, éd. cit., v. 2152-2155).
} 
Dans le Dit d'Entendement ${ }^{44}$ (1295-1300) de Jean de Condé, Renart, gouverneur et maître de l'Hôtel royal (v. 778), dirige, comme chez Rutebeuf, les affaires d'État et règne sur le pays :

- Amis, dist il, au jour d'ui court

Renars et regne en mainte court (v. 1028-1029)

Connoistre pues certainement

Que Renars queurt par tout le monde ;

Tant come il dure a la reonde

A espandu sa renardie (v. 1060-1063)

Ses fils, l'un Jacobin, l'autre Cordelier, occupent de bonnes places à la cour aux côtés d'Isengrin et du singe Martin ${ }^{45}$. Les personnages sont des symboles :

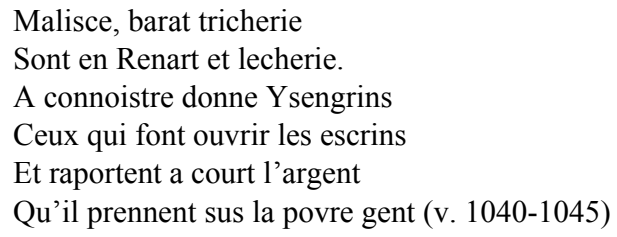

Dans l'univers féodalisé de Renart le Nouvel ${ }^{46}$, Renart réalise une synthèse de tous les maux. Figura diaboli, il triomphe à la fin du roman : Renart le Nouvel se clôt sur son apogée ; Fortune lui suggère de venir siéger pour l'éternité au sommet de sa roue qui ne tournera plus (v. 7692-7715).

A icest mot Renars monta

Sour la roe tous courounés,

D’Orguel et de Guile adestrés. (v. 7716-7718)

Et, sur la grande enluminure présente au recto du dernier feuillet du manuscrit fr. 1581 de la Bibliothèque nationale de France, Renart est représenté en majesté, assis sur un trône au sommet de la roue de Fortune. Le goupil n'est plus livré aux incessantes variations de la vie, assujetti à la quête de liberté ou de nourriture comme dans le Roman de Renart. Son image se fixe, comme ici sur la roue de Fortune, avec un sens unique; son évolution montre aussi que son nom seul désigne désormais la bête, toute entière symbole du Mal.

\footnotetext{
${ }^{44}$ Le Dit d'Entendement est édité par A. Scheler dans Dits et contes de Baudouin de Condé et de son fils Jean de Condé, Bruxelles, V. Devaux, 1866-1867, T. 3, pièce 38, p. 49-95; il est partiellement édité dans Le Roman de Renart, éd. cit., p. 897-904.

${ }^{45}$ Le singe est une autre image du Mal dans l'imaginaire médiéval; il est par exemple assimilé au diable dans le Physiologus.

${ }^{46}$ Renart le Nouvel est composé par Jacquemart Gielée en 1289 ; il a été publié d'après le manuscrit La Vallière par H. Roussel, Paris, Picard, "Société des anciens textes français ", 1961.
} 
À cela correspond en effet un changement des motifs. Les textes ne se caractérisent plus par ce que l'on a appelé pour le Roman de Renart une épopée de la faim et ils perdent le jeu si fréquent dans le Roman entre anthropomorphisme et zoomorphisme qui définissait un univers de fantaisie ${ }^{47}$ constamment en déséquilibre. Partout, en particulier dans Renart le Nouvel qui privilégie les liens de féodalité, une bien faible part est accordée à l'animalité du personnage. Ainsi, en entrant dans l'allégorie, l'animal, son nom seul «Renard/t», est traité comme le signe du Mal. Les modalités d'écriture varient également et s'adaptent à la moralisation des récits. Alors que Philippe de Novare imitait encore les branches du Roman de Renart ${ }^{48}$, devenus symboliques et moraux $^{49}$, les épigones adoptent généralement une forme différente. Les œuvres de Rutebeuf et de Jean de Condé sont des dits, la forme est plus didactique et même satirique. Le Dit d'Entendement est même allégorique. Le long roman du Couronnement de Renart comme Renart le Nouvel prolongent cette veine. Le cas de Renart le Contrefait $t^{50}$ est particulier. Écrit pendant le deuxième tiers du XIV ${ }^{\mathrm{e}}$ siècle, le roman est constitué par une série de branches rappelant plusieurs épisodes du Roman de Renart (la confession au milan, le puits, le songe de Chantecler...). Mais l'auteur, un clerc de Troyes, intercale au milieu des épisodes de longs développements narratifs, des exempla, de violentes critiques contre le haut clergé et la noblesse.

L'image du renard se fixe : il est le Mal, l'agent du diable sur la terre et il répand sa renardie sur les $\mathrm{XIII}^{\mathrm{e}}$ et $\mathrm{XIV}^{\mathrm{e}}$ siècles. Pour Rutebeuf comme pour ses contemporains, l'animal constitue le moyen de développer une satire violente contre les hypocrites et en particulier contre les ordres mendiants. L'image est un artifice rhétorique pour dénoncer les déguisements et le monde bestourné.

\section{L'engin et l'art de Rutebeuf}

L'auteur de Renart le Contrefait a pris le masque de Renart pour dénoncer les vices de son siècle :

Pour dire par escrit couvert

Ce qu'il n'osoit dire en appert. (v. 121-122)

Rutebeuf, lui, a pris son art: le bestournement est le principe même de la satire. Le poète a changé les hommes en bêtes pour pouvoir dénoncer leurs pratiques, il les a, littéralement, bestournés: dans Renart le Bestourné, il les a dotés de visages

\footnotetext{
${ }^{47}$ L'expression est empruntée à R. Bellon, «Renart le Nouvel, réemploi et innovation, de l'épopée animale au poème allégorique », Cahier Robinson, 2004, p. 27-41.

${ }^{48}$ Selon G. Paris et J. Flinn, on aurait pu croire cette branche authentiquement renardienne. Voir le chapitre de J. Flinn intitulé «Philippe de Novare et le Roman de Renart», dans Le Roman de Renart dans la littérature française et les littératures étrangères de Moyen Age, Paris, PUF, 1963, p. 171.

${ }^{49}$ Le manuscrit $C$ de Renart le Nouvel débute par exemple par cette rubrique : «Ci coumence le roumans du petit Renart de moralité ». La variante est répertoriée par H. Roussel dans Renart le Nouvel, éd. cit., p. 13.

${ }^{50}$ Le Roman de Renart le Contrefait est édité par G. Raynaud et H. Lemaître, Paris, Champion, 1914.
} 
animaux, comme ailleurs il se sert d'allégories pour dénoncer les vices et lever les masques, dans La Voix d'Humilité ou dans La Leçon sur Hypocrisie et Humilité. L'écriture de Rutebeuf souhaite établir à son tour un nouvel ordre des choses, revenir en arrière, vers le passé glorieux et chevaleresque de Charlemagne, de Roland et d'Olivier ${ }^{51}$, des croisades $^{52}$. La fin de Renart le bestourné donne cette leçon d'un possible renversement de Renart et de ses acolytes. Car «Tot pert qui tot tient» (v. 153). Le poète guette le moment du Jugement dernier et de l'Apocalypse, l'arrivée de l'Once:

La choze gist en teil endroit

Que chacune beste vorroit

Que venist l'Once. (v. 155-157)

L'esthétique du poète repose elle-même sur un art du retournement. Rutebeuf s'amuse régulièrement à renverser les motifs de la poésie médiévale: il manipule, comme on l'a vu, la tradition du Roman de Renart, il parodie aussi des motifs de la poésie courtoise. La reverdie déjà réécrite dans La Voix d'Humilité (v. 1-10) est en tout point inversée au début de La Griesche d'hiver ou de La Leçon sur Hypocrisie et Humilité :

Au tens que les cornoilles braient,

Qui por la froidure s'esmaient

Qui seur les cors lor vient errant,

Qu'eles vont ces noiz enterrant

Et s'en garnissent por l'iver,

Qu'en terre sunt entrei li ver... (v. 1-6)

De même, Rutebeuf remplace le dénuement et l'indigence ${ }^{53}$ par la plus grande richesse poétique, dans une œuvre qui décline de multiples formes, où le dit tend à la fois vers le fabliau, le récit allégorique, la branche.

L'entreprise de Rutebeuf s'appuie sur ces phénomènes de satire et de parodie mais aussi sur la manipulation du langage. Sa rhétorique repose amplement sur les syllepses et les homophonies, les jeux d'annominatio qui renforcent certains rapprochements et densifient le sens. Le tercet coué utilisé dans Renart le Bestourné révèle encore la virtuosité et l'art du poète qui par son travail des sons et des signes s'attache à dénoncer les fausses valeurs et les vices. Ainsi, la poétique de Rutebeuf est essentiellement ironique: elle procède par détour et par dédoublement des significations pour dénoncer les pièges et les masques du langage et des signes. Elle

\footnotetext{
${ }^{51}$ Voir en particulier La Complainte de Sainte Eglise : «Ce Charles fust en France ou ce i fust Rollanz, / Ne peüssent contre aux ne Yaumons n'Agolans », v. 15-16.

${ }^{52}$ Voir Poèmes de l'infortune et autres poèmes édités par J. Dufournet, Paris, Gallimard, «Poésie », 1986.

${ }^{53}$ Voir en particulier Poèmes de l'infortune, ibid. Voir aussi l'article de R. Dragonetti : «Rutebeuf: les poèmes de la «griesche»», La Musique et les lettres : études de littérature, op. cit., p. 435-462.
} 
agit par inversion et antiphrase, par hyperbole ou au contraire par litote et ainsi révèle un sens nouveau. Le bestournement est un procédé de l'ironie.

Mais cet art de l'inversion qui doit dénoncer les mendiants rapproche dangereusement le poète de ceux qu'il dénonce et Rutebeuf trouve dans sa rhétorique un plaisir inquiétant. Comme les mendiants, par exemple dans La Leçon sur Hypocrisie et Humilité, Rutebeuf se masque et se déguise :

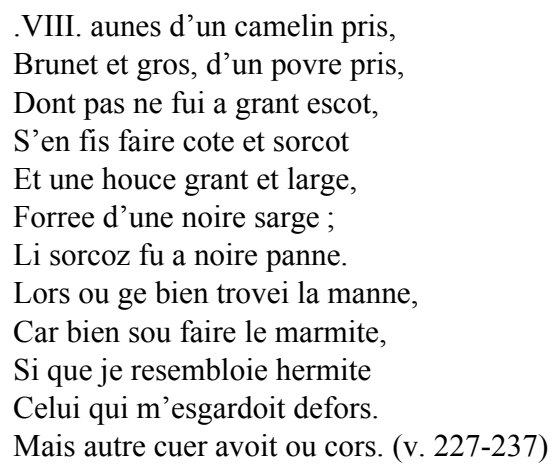

De façon plus régulière et plus emblématique, le nom du poète est lui-même un signe manipulé et animalisé. «Rutebeuf » est un calembour composé de « rude » et de «bœuf " $^{54}$ - à moins que « rude », régulièrement graphié « ruste» signifie « vigoureux, impétueux ». Le poète serait alors, comme le dit J. Dufournet, «un taureau déguisé en bœuf $»^{55}$. Le nom est fabriqué : Rutebeuf invente une fiction qui définit son œuvre et qui caractérise son labeur; il se décline aussi selon une pratique jouissive de la langue poétique. Surtout, le pseudonyme est un masque : la fiction a à voir avec le mensonge et régulièrement, l'adverbe «rudement» rime avec le présent du verbe "mentir», dans La Vie de sainte Elysabel (v. 1999-2000) comme dans Le Miracle du Sacristain :

Rutebuez huevre rudement,

Souvent en sa rudesce ment. (759-760)

Rutebeuf joue; les mots sont pris dans le tourbillon, dans les syllepses, les assonances, les rimes. L'écriture provoque une ivresse de sons qui perd le lecteur et le détourne aussi du sens. Ainsi, la parole poétique de Rutebeuf, qui ne cesse d'osciller entre la vérité et le masque, entre la révélation et le jeu, est essentiellement ambiguë. Car la jouissance de la langue, des arts du langage a partie liée avec la séduction. La verve du poète semble contaminée, bestournée, par cela même qu'elle voulait dénoncer: les faux-semblants, les masques, la séduction et la diabolie. Comme le souligne R. Dragonetti dans sa préface aux études rassemblées de

\footnotetext{
${ }^{54}$ Voir en particulier La Leçon sur Hypocrisie et Humilité, v. 44-46 ou La Vie de saint Marie l'Égypyienne, v. 1301-1302.

${ }^{55}$ J. Dufournet, Préface aux Poèmes de l'infortune, op. cit., p. 8.
} 
J. Dufournet, les arts du langage n'échappent pas à l'emprise de la ruse et de la duplicités6.

La parole de Rutebeuf repose donc sur un rapport rusé avec le monde et avec l'écriture, sur un rapport renardien avec sa production. Rutebeuf semble lui-même posséder «engin et art» et sa langue exerce, comme celle de Renart, une fascination poétique. Elle dénonce et démasque, elle séduit et elle trompe, elle ment aussi, comme les mendiants. Elle est même «mauvaise », selon le Dit du mensonge :

Maugrei toutes les langues males

Et la Rutebuef douz premiers (v. 82-83) Bernard :

La manière poétique de Rutebeuf trouve un écho dans les paroles de saint

La langue s'appelle langue parce qu'elle lèche. Elle lèche en flattant, elle mord en médisant, elle tue en mentant. Elle lie et ne peut être liée : elle est glissante et on ne peut la tenir : mais elle s'échappe et elle est trompée. Elle glisse comme l'anguille, elle pique comme une flèche... elle est douce et rusée, large et prête à épuiser le bien et à mélanger le mal. «Qui garde sa langue garde son âme : parce que la mort et la vie sont au pouvoir de la langue $»^{57}$.

Le renard de Rutebeuf est un animal fourbe et rusé; il est aussi un représentant du diable sur terre, une image sombre des mendiants et des hypocrites que le poète n'a de cesse de dénoncer. Mais par ses satires et sa rhétorique, Rutebeuf s'est rendu secrètement complice de Renart; lui-même s'est fait hypocrite et s'est essayé à l'art de la séduction et de la déception : la renardie.

Les derniers vers de La Repentance Rutebeuf soulignent toutefois l'échec du poète qui n'a pas égalé l'art de Renart. Dans ce monde "qui se depart», le repentir sonne comme un renoncement à la poésie :

Je cuidai engignier Renart :

Or n'i vallent enging ne art,

Qu'asseür [est] en son palais.

Por cest siecle qui se depart

Me couvient partir d'autre part.

Qui que l'envie, je le lés. (v. 79-84)

\footnotetext{
${ }^{56}$ Dans L'Univers de Rutebeuf, op. cit., p. 12-18.

${ }^{57}$ Saint Bernard, Traité de la maison intérieure, Chap. XXVII « Des défauts et des abus de la langue, du chant, du jugement, etc... », CEuvres complètes, Paris, Louis Vivès, 1867, vol. 6, p. 33 .
} 
Mais la leçon de cette fin est aussi ambiguë : comme le goupil, Rutebeuf contrefait peut-être le mort pour continuer à écrire ${ }^{58}$. Pourtant, Rutebeuf se tourne bien vers l'espoir que représentent Dieu et la Vierge. L'appel de la transcendance trace une nouvelle voie poétique; la voix de Rutebeuf parvient à s'extraire de la «lobe» renardienne.

Aurélie Barre Université de Lyon 3

${ }^{58}$ Sur ce dit, voir l'étude de J. Dufournet: «La Repentance Rutebeuf ou le mot de la fin», L'Univers de Rutebeuf, op. cit., p. 102-118. 